International Electronic Journal of Geometry

Volume 8 No. 2 PP. 82-96 (2015) (C)IEJG

\title{
THE ISOMETRY GROUP OF CHINESE CHECKER SPACE
}

\author{
ÖZCAN GELIŞGEN AND RÜSTEM KAYA
}

(Communicated by Yusuf YAYLI )

\begin{abstract}
In this article, we, firstly, find that the spheres in the Chinese Checkers space are deltoidal icositetrahedrons. Then we show that the group of isometries of the 3-dimensional space with respect to Chinese Checkers metric is the semi-direct product of deltoidal icositetrahedron group $G(D)$ and $T(3)$, where $G(D)$ is the (Euclidean) symmetry group of the deltoidal icositetrahedron and $T(3)$ is the group of all translations of the 3-dimensional space.
\end{abstract}

\section{INTRODUCTION}

Convex sets plays an important role in determination of the group of isometries of certain geometries. Minkowski geometry is a non-Euclidean geometry in a finite number of dimensions that is different from elliptic and hyperbolic geometry (and from the Minkowskian geometry of space-time). In a Minkowski geometry the linear structure is the same as the Euclidean one but distance is not uniform in all directions. Instead of the usual sphere in Euclidean space, the unit ball is a certain symmetric closed convex set (Thompson [16]). Each of the geometries induced by maximum, taxicab, Chinese Checkers, $\alpha_{i}$ and $\lambda$-distances is one of the geometry of this type ( [11], [13], [7], [14], [10], [8]).

Chinese Checkers geometry proposed by Krause [[11], p.65] asking the question of how to develop a metric which would be similar to the movement by playing Chinese Checkers. Later Chen [4] gave such a metric for the coordinate plane. CC-plane geometry has been studied and improved by some mathematicians, (for some references see So [15] and Colakoglu-Kaya [5]). Three dimensional CC-space has been introduced and studied analytically by Gelisgen-Kaya-Ozcan [6]. In this work which is motivated by [9] and [8], we extend the result of CC-plane to three dimensional CC-space and determine the group of isometries of it.

\section{The CC-Spheres and Preliminaries}

The Chinese Checkers 3-dimensional space $\mathbb{R}_{c}^{3}$ is almost the same as the Euclidean analytical 3 -dimensional space $\mathbb{R}^{3}$. The points, lines and planes are the

Date: Received: December 05, 2012 and Accepted: May 18, 2015.

2010 Mathematics Subject Classification. 51B20, 51F99, 51K05, 51K99, 51N25.

Key words and phrases. Group, isometry, metric, Chinese Checkers metric, Chinese Checkers geometry. 
same, and the angles are measured in the same way, but the distance function is different. CC-metric for 3-dimensional analytical space was introduced in [6]. CC-metric is defined using the following distance function

$$
d_{c}(A, B)=d_{L}(A, B)+(\sqrt{2}-1) d_{S}(A, B)
$$

where

and

$$
d_{L}(A, B)=\max \left\{\left|x_{1}-x_{2}\right|,\left|y_{1}-y_{2}\right|,\left|z_{1}-z_{2}\right|\right\}
$$

$$
d_{S}(A, B)=\min \left\{\left|x_{1}-x_{2}\right|+\left|y_{1}-y_{2}\right|,\left|x_{1}-x_{2}\right|+\left|z_{1}-z_{2}\right|,\left|y_{1}-y_{2}\right|+\left|z_{1}-z_{2}\right|\right\}
$$

where $A=\left(x_{1}, y_{1}, z_{1}\right), B=\left(x_{2}, y_{2}, z_{2}\right)$. According to definition of $d_{c}$-distance the shortest path between the points $A$ and $B$ is the union of three line segments which one of them is parallel to a coordinate axes and other line segments each making $\pi / 4$ angle with one of other coordinate axes as shown in Fig. 1 . Thus, the shortest $d_{c}$-distance between $A$ and $B$ is sum of the Euclidean lengths of such three line segments.

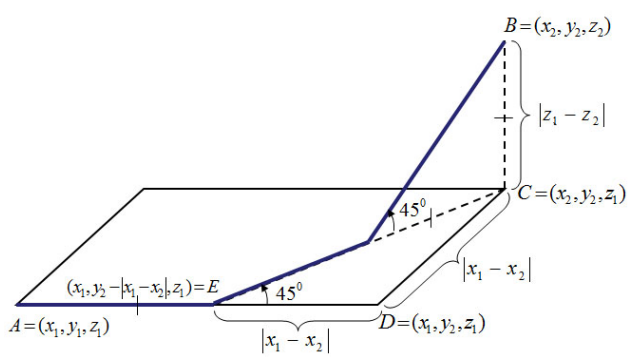

Figure 1(a)

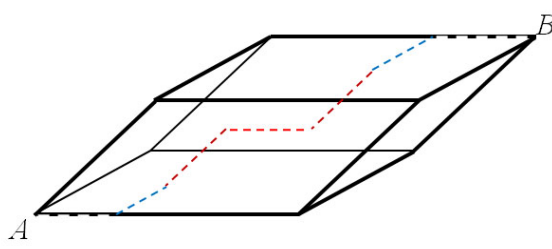

Figure 1(b)

Figure 1(a) and Figure 1(b) illustrate $\mathrm{CC}$ way from $A$ to $B$ in the case $\left|y_{1}-y_{2}\right| \geq\left|x_{1}-x_{2}\right| \geq\left|z_{1}-z_{2}\right|$ and some of ways from $A$ to $B$, respectively.

Proposition 2.1. A chinese Checkers sphere is a deltoidal icositetrahedron.

Proof. A Chinese Checkers sphere (ball) with center $O$ and radius $r$ in $\mathbb{R}_{c}^{3}$ is the set of points $(x, y, z)$ in the 3 -dimensional space satisfying the equation

$$
\max \{|x|,|y|,|z|\}+(\sqrt{2}-1) \min \{|x|+|y|,|x|+|z|,|y|+|z|\}=r
$$

which is a polyhedra which has 24 -faces with vertices

$$
\begin{aligned}
& A_{1}=(r, 0,0), A_{2}=(r / \sqrt{2}, r / \sqrt{2}, 0), A_{3}=(0, r, 0), A_{4}=(-r / \sqrt{2}, r / \sqrt{2}, 0), \\
& A_{5}=(-r, 0,0), A_{6}=(-r / \sqrt{2},-r / \sqrt{2}, 0), A_{7}=(0,-r, 0), A_{8}=(r / \sqrt{2},-r / \sqrt{2}, 0), \\
& A_{9}=(r / \sqrt{2}, 0, r / \sqrt{2}), A_{10}=(0, r / \sqrt{2}, r / \sqrt{2}), A_{11}=(-r / \sqrt{2}, 0, r / \sqrt{2}), \\
& A_{12}=(0,-r / \sqrt{2}, r / \sqrt{2}), A_{13}=(r / \sqrt{2}, 0,-r / \sqrt{2}), A_{14}=(0, r / \sqrt{2},-r / \sqrt{2}), \\
& A_{15}=(-r / \sqrt{2}, 0,-r / \sqrt{2}), A_{16}=(0,-r / \sqrt{2},-r / \sqrt{2}), A_{17}=(0,0, r), A_{18}=(0,0,-r), \\
& A_{19}=(r /(2 \sqrt{2}-1), r /(2 \sqrt{2}-1), r /(2 \sqrt{2}-1)), A_{20}=(-r /(2 \sqrt{2}-1), r /(2 \sqrt{2}-1), r /(2 \sqrt{2}-1)), \\
& A_{21}=(-r /(2 \sqrt{2}-1),-r /(2 \sqrt{2}-1), r /(2 \sqrt{2}-1)), A_{22}=(r /(2 \sqrt{2}-1),-r /(2 \sqrt{2}-1), r /(2 \sqrt{2}-1)), \\
& A_{23}=(r /(2 \sqrt{2}-1), r /(2 \sqrt{2}-1),-r /(2 \sqrt{2}-1)), A_{24}=(-r /(2 \sqrt{2}-1), r /(2 \sqrt{2}-1),-r /(2 \sqrt{2}-1)), \\
& A_{25}=(-r /(2 \sqrt{2}-1),-r /(2 \sqrt{2}-1),-r /(2 \sqrt{2}-1)), A_{26}=(r /(2 \sqrt{2}-1),-r /(2 \sqrt{2}-1),-r /(2 \sqrt{2}-1))
\end{aligned}
$$


as shown in Fig. 2.

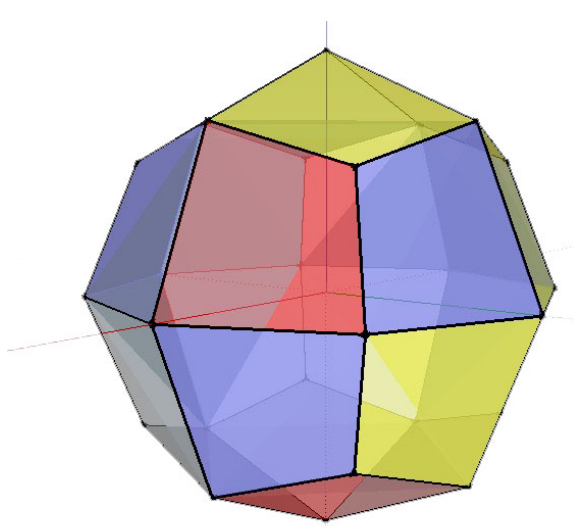

Figure 2(a)

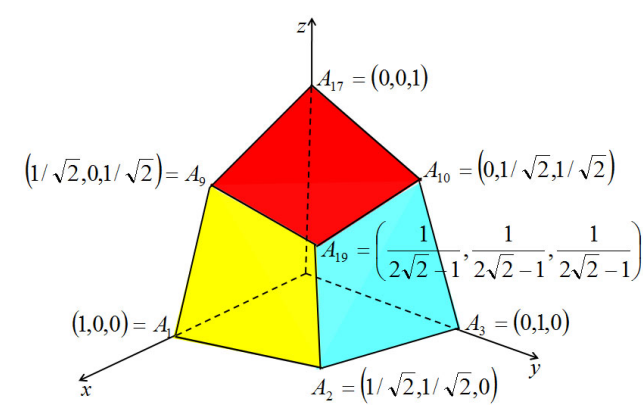

Figure 2(b)

Figure 2(a) and Figure 2(b) show the graph of the $C C$-sphere and graph of the unit $C C$-sphere in the first octant, respectively.

CC-unit sphere has 24 deltoidal faces each of which is the part of plane having one of the following equations

$$
\begin{aligned}
& \mp x \mp(\sqrt{2}-1)(y \mp z) \pm 1=0 \\
& \mp y \mp(\sqrt{2}-1)(x \mp z) \pm 1=0 \\
& \mp z \mp(\sqrt{2}-1)(x \mp y) \pm 1=0 .
\end{aligned}
$$

In order to obtain the faces of CC-unit sphere, it is enough to take $x, y, z \in[-1,1]$ in the above equations. It is clear from Figure 2, CC-unit sphere consist of 24 (twenty-four) deltoids or kites. The short and long edges of each deltoid are in the ratio $1,00: 1,29$.

It is clear from the above information that a CC-sphere is a polyhedra with 24 faces, 48 edges and 26 vertices. This polyhedra is neither a regular nor a semiregular solid but satisfies the Euler relation $V-E+F=2$. Also this polyhedra is a Catalan solid. Each of 24 edges have length $\sqrt{2-\sqrt{2}}$ and the other 24 have length $(\sqrt{208-136 \sqrt{2}}) / 14$ Euclidean unit. Each of 8 vertices joins exactly 3 deltoids and 3 edges; and the remaining 18 vertices join 4 deltoids and 4 edges.

The CC-sphere is one of the Catalan solids and its special name is Deltoidal icositetrahedron. A Catalan solid is a dual polyhedron to an Archimedean solid. The Catalan solids are named for the Belgian mathematician, Eugène Catalan, who first described them in 1865 .

The Catalan solids are all convex. They are face-transitive but not vertextransitive. This is because the dual Archimedean solids are vertex-transitive and not face-transitive. Note that unlike Platonic solids and Archimedean solids, the faces of Catalan solids are not regular polygons. However, the vertex figures of Catalan solids are regular, and they have constant dihedral angles. A deltoidal icositetrahedron is a Catalan solid which looks a bit like an overinflated cube. Its 
dual polyhedron is the rhombicuboctahedron. The deltoidal icositetrahedron is topologically identical to a cube which has all of its edges bisected.

We take the following Lemma from Gelişgen-Kaya-Ozcan [6], which will be useful to determine reflections in $\mathbb{R}_{c}^{3}$.

Lemma 2.1. Let $l$ be the line through the points $A=\left(x_{1}, y_{1}, z_{1}\right)$ and $B=\left(x_{2}, y_{2}, z_{2}\right)$ in the analytical 3-dimensional space and $d_{E}$ denote the Euclidean metric. If $l$ has direction vector $(p, q, r)$, then

$$
d_{c}(A, B)=\mu_{A B} d_{E}(A, B)
$$

where

$$
\mu_{A B}=\frac{\max \{|p|,|q|,|r|\}+(\sqrt{2}-1) \min \{|p|+|q|,|p|+|r|,|q|+|r|\}}{\sqrt{p^{2}+q^{2}+r^{2}}} .
$$

Proof. Equation of $l$ gives us $x_{1}-x_{2}=\lambda p, y_{1}-y_{2}=\lambda q, z_{1}-z_{2}=\lambda r, r \in \mathbb{R}$. Thus, $d_{c}(A, B)=|\lambda|(\max \{|p|,|q|,|r|\}+(\sqrt{2}-1) \min \{|p|+|q|,|p|+|r|,|q|+|r|\})$ and $d_{E}(A, B)=|\lambda| \sqrt{p^{2}+q^{2}+r^{2}}$ which implies the required result.

The above lemma says that $d_{c}$-distance along any line is some positive constant multiple of Euclidean distance along same line. Thus, one can immediately state the following corollaries:

Corollary 2.1. If $A, B$ and $X$ are any three collinear points in $\mathbb{R}^{3}$, then $d_{E}(A, X)=d_{E}(B, X)$ if and only if $d_{c}(A, X)=d_{c}(B, X)$.

Corollary 2.2. If $A, B$ and $X$ are any three distinct collinear points in the real 3-dimensional space, then

$$
d_{c}(X, A) / d_{c}(X, B)=d_{E}(X, A) / d_{E}(X, B) .
$$

That is, the ratios of the Euclidean and $d_{c}$-distances along a line are the same.

In the remaining part of this work, we will study the isometries of $\mathbb{R}_{c}^{3}$, and determine its group of isometries.

\section{Isometries of The Chinese Checkers Space $\left(\mathbb{R}_{c}^{3}\right)$}

One of the basic problems in geometric investigations for a given space $S$ with a metric $d$ is to describe the group $G$ of isometries. If $S$ is the Euclidean 3-dimensional space with the usual metric, then it is well known that $G$ consists of all of translations, rotations, reflections, glide reflections, rotary reflection and screw of the 3 -dimensional space.

It is known that for the Euclidean 3-dimensional space $G=E(3)$ is the semidirect product of its two subgroups $O(3)$ (the orthogonal group) and $T(3)$, where $O(3)$ is the symmetry group of the unit sphere and $T(3)$ (the translation group) consists of all translations of 3-dimensional space.

Throughout the article we use the following definitions needed, which adopted from Martin [12]:

A transformation on Chinese Checkers 3-space $\mathbb{R}_{c}^{3}$ is one-to-one correspondence from the set of points in space onto itself. Transformation $\alpha$ is called an isometry if $d_{c}(X, Y)=d_{c}(\alpha(X), \alpha(Y))$ for all points $X$ and $Y$. The identity $i$ is defined by 
$i(X)=X$ for every point $X$. Isometry $\alpha$ is a symmetry for set of points if $\alpha$ fixes that set of points.

If $\Delta$ is a plane, then the reflection $\sigma_{\Delta}$ is the mapping on the points in $\mathbb{R}_{c}$ such that $\sigma_{\Delta}(X)=X$ if point $X$ is on $\Delta$ and $\sigma_{\Delta}(X)=Y$ if point $X$ is off $\Delta$ and plane $\Delta$ is perpendicular bisector of the line segment $X Y$.

If two planes $\Gamma$ and $\Delta$ intersect at line $l$, then $\sigma_{\Delta} \sigma_{\Gamma}$ is called a rotation about axis $l$.

If $\Gamma$ and $\Delta$ are two intersecting planes each perpendicular to plane $\Pi$, then $\sigma_{\Pi} \sigma_{\Delta} \sigma_{\Gamma}$ is called a rotary reflection (a rotatory reflection) about the point common to $\Gamma, \Delta$ and $\Pi$. If $M$ is a point, the inversion $\sigma_{M}$ about $M$ is the transformation such that $\sigma_{M}(X)=Y$ for all points $X$ where $M$ is the midpoint of $X$ and $Y . \sigma_{M}$ is sometimes called a point reflection.

If $\Gamma$ and $\Delta$ are two intersecting planes and $M$ is a point common to $\Gamma, \Delta$, then $\sigma_{M} \sigma_{\Delta} \sigma_{\Gamma}$ is called a rotary inversion.

If planes $\Gamma$ and $\Delta$ are parallel, that is, $\Gamma \| \Delta$, then $\sigma_{\Delta} \sigma_{\Gamma}$ is a translation along the common perpendicular lines to planes $\Gamma$ and $\Delta$.

Since the CC-space geometry is the study of Euclidean points, lines, planes and angles in $\mathbb{R}^{3}$, an isometry of $\mathbb{R}_{c}^{3}$ is therefore an isometry of the real space with respect to the $d_{c}$ metric.

Proposition 3.1. Every Euclidean translation of $\mathbb{R}^{3}$ is an isometry of $\mathbb{R}_{c}^{3}$.

Proof. Let $T_{A}: \mathbb{R}_{c}^{3} \rightarrow \mathbb{R}_{c}^{3}$ such that $T_{A}(X)=A+X$ be translation as in the real 3-dimensional space $\mathbb{R}^{3}$, where $A=\left(a_{1}, a_{2}, a_{3}\right)$ and $X=\left(x_{1}, y_{1}, z_{1}\right) \in \mathbb{R}_{c}$. For $X=\left(x_{1}, y_{1}, z_{1}\right)$ and $Y=\left(x_{2}, y_{2}, z_{2}\right) \in \mathbb{R}_{c}$, we have

$$
\begin{aligned}
d_{c}\left(T_{A}(X)\right. & \left., T_{A}(Y)\right)=\max \left\{\left|a_{1}+x_{1}-a_{1}-x_{2}\right|,\left|a_{2}+y_{1}-a_{2}-y_{2}\right|,\left|a_{3}+z_{1}-a_{3}-z_{2}\right|\right\} \\
& +(\sqrt{2}-1) \min \left\{\left|a_{1}+x_{1}-a_{1}-x_{2}\right|+\left|a_{2}+y_{1}-a_{2}-y_{2}\right|,\right. \\
& \left.\left|a_{1}+x_{1}-a_{1}-x_{2}\right|+\left|a_{3}+z_{1}-a_{3}-z_{2}\right|,\left|a_{2}+y_{1}-a_{2}-y_{2}\right|+\left|a_{3}+z_{1}-a_{3}-z_{2}\right|\right\} \\
& =\max \left\{\left|x_{1}-x_{2}\right|,\left|y_{1}-y_{2}\right|,\left|z_{1}-z_{2}\right|\right\}+(\sqrt{2}-1) \\
& \min \left\{\left|x_{1}-x_{2}\right|+\left|y_{1}-y_{2}\right|,\left|x_{1}-x_{2}\right|+\left|z_{1}-z_{2}\right|,\left|y_{1}-y_{2}\right|+\left|z_{1}-z_{2}\right|\right\} \\
& =d_{c}(X, Y) .
\end{aligned}
$$

That is, $T_{A}$ is an isometry.

Notice that it is enough to consider the planes passing through the origin to find the rotations and the reflections in $\mathbb{R}_{c}^{3}$ by Proposition 3.1. The following lemma helps to determine the reflections which preserves the distance in $\mathbb{R}_{c}^{3}$.

Lemma 3.1. A reflection about the plane $\Delta: a x+b y+c z=0$ in $\mathbb{R}_{c}^{3}$ is an isometry if and only if $(a, b, c)$ is parallel to an element the set of vectors

$$
D=\{(1,0,0),(0,1,0),(0,0,1),( \pm 1,1,0),(1,0, \pm 1),(0,1, \pm 1)\} .
$$

Proof. Without loss of the generality consider the Euclidean reflection $\sigma_{\Delta}$ about the plane $a x+b y+c z=0$ with the unit normal vector $(a, b, c), \sigma_{\Delta}: \mathbb{R}_{c}^{3} \rightarrow \mathbb{R}_{c}^{3}$ such that $\sigma_{\Delta}(x, y, z)=$

$$
\left(\left(1-2 a^{2}\right) x-2 a b y-2 a c z,-2 a b x+\left(1-2 b^{2}\right) y-2 b c z,-2 a c x-2 b c y+\left(1-2 c^{2}\right) z\right) \text {. }
$$

Since vector set $\left\{E_{1}=(1,0,0), E_{2}=(0,1,0), E_{3}=(0,0,1)\right\}$ is a base of $\mathbb{R}^{3}$, a reflection which preserves this base must be an isometry. So it is sufficient to determine the reflections which preserves vectors of this base. Applying Euclidean 
reflection to vectors of the base, one can get

$$
\begin{aligned}
& \sigma_{\Delta}(1,0,0)=\left(1-2 a^{2},-2 a b,-2 a c\right) \\
& \sigma_{\Delta}(0,1,0)=\left(-2 a b, 1-2 b^{2},-2 b c\right) \\
& \sigma_{\Delta}(0,0,1)=\left(-2 a c,-2 b c, 1-2 c^{2}\right) .
\end{aligned}
$$

Clearly $d_{c}\left(O, E_{1}\right)=d_{c}\left(O, E_{2}\right)=d_{c}\left(O, E_{3}\right)=1$. If a reflection preserves $d_{c^{-}}$ distance, we must look for $a, b, c$ which implies

$d_{c}\left(\sigma_{\Delta}(O), \sigma_{\Delta}\left(E_{1}\right)\right)=d_{c}\left(\sigma_{\Delta}(O), \sigma_{\Delta}\left(E_{2}\right)\right)=d_{c}\left(\sigma_{\Delta}(O), \sigma_{\Delta}\left(E_{3}\right)\right)=1$.

Thus,

if and only if

$$
\begin{aligned}
& d_{c}\left(\sigma_{\Delta}(O), \sigma_{\Delta}\left(E_{1}\right)\right)=1 \\
& d_{c}\left(\sigma_{\Delta}(O), \sigma_{\Delta}\left(E_{2}\right)\right)=1 \\
& d_{c}\left(\sigma_{\Delta}(O), \sigma_{\Delta}\left(E_{3}\right)\right)=1
\end{aligned}
$$

$$
\begin{gathered}
\max \left\{\left|2 a^{2}-1\right|,|2 a b|,|2 a c|\right\}+(\sqrt{2}-1) \\
\min \left\{\left|2 a^{2}-1\right|+|2 a b|,\left|2 a^{2}-1\right|+|2 a c|,|2 a b|+|2 a c|\right\}=1 \\
\max \left\{|2 a b|,\left|2 b^{2}-1\right|,|2 b c|\right\}+(\sqrt{2}-1) \\
\min \left\{|2 a b|+\left|2 b^{2}-1\right|,|2 a b|+|2 b c|,\left|2 b^{2}-1\right|+|2 b c|\right\}=1 \\
\max \left\{|2 a c|,|2 b c|,\left|2 c^{2}-1\right|\right\}+(\sqrt{2}-1) \\
\min \left\{|2 a c|+|2 b c|,|2 a c|+\left|2 c^{2}-1\right|,|2 b c|+\left|2 c^{2}-1\right|\right\}=1 .
\end{gathered}
$$

Now, one can solve this system of equations and obtain the solutions

$$
(1,0,0),(0,1,0),(0,0,1),\left( \pm \frac{1}{\sqrt{2}}, \frac{1}{\sqrt{2}}, 0\right),\left(\frac{1}{\sqrt{2}}, 0, \pm \frac{1}{\sqrt{2}}\right) \text { and }\left(0, \frac{1}{\sqrt{2}}, \pm \frac{1}{\sqrt{2}}\right) .
$$

Conversely, if $\left(p_{1}, q_{1}, r_{1}\right)$ and $\left(p_{2}, q_{2}, r_{2}\right)$ are the direction vectors of the lines $O X$ and $O Y$, where $\sigma_{\Delta}(X)=Y$, then $d_{E}(O, X)=d_{E}(O, Y), d_{c}(O, X)=\mu_{O X} d_{E}(O, X)$, $d_{c}(O, Y)=\mu_{O Y} d_{E}(O, Y)=\mu_{O Y} d_{E}(O, X)$, where

$$
\mu_{A B}=\frac{\max \{|p|,|q|,|r|\}+(\sqrt{2}-1) \min \{|p|+|q|,|p|+|r|,|q|+|r|\}}{\sqrt{p^{2}+q^{2}+r^{2}}}
$$

by Lemma 2.1. Now it is easy to check that $\mu_{O X}=\mu_{O Y}$ for all possible cases in the following table, which implies $d_{c}(O, X)=d_{c}(O, Y)$.

\begin{tabular}{c|c}
$\Delta$ & $\left(p_{2}, q_{2}, r_{2}\right)$ \\
\hline$x=0$ & $\left(-p_{1}, q_{1}, r_{1}\right)$ \\
\hline$y=0$ & $\left(p_{1},-q_{1}, r_{1}\right)$ \\
\hline$z=0$ & $\left(p_{1}, q_{1},-r_{1}\right)$ \\
\hline$x+y=0$ & $\left(-2 q_{1},-2 p_{1}, 2 r_{1}\right)$ \\
\hline$x-y=0$ & $\left(2 q_{1}, 2 p_{1}, 2 r_{1}\right)$ \\
\hline
\end{tabular}

\begin{tabular}{c|c}
$\Delta$ & $\left(p_{2}, q_{2}, r_{2}\right)$ \\
\hline$x+z=0$ & $\left(-2 r_{1}, 2 q_{1},-2 p_{1}\right)$ \\
\hline$x-z=0$ & $\left(2 r_{1}, 2 q_{1}, 2 p_{1}\right)$ \\
\hline$y+z=0$ & $\left(2 p_{1},-2 r_{1},-2 q_{1}\right)$ \\
\hline$y-z=0$ & $\left(2 p_{1}, 2 r_{1}, 2 q_{1}\right)$ \\
\hline
\end{tabular}

Thus, the set $S_{c}$ of isometric reflections about the planes passing through the origin consists of the nine Euclidean reflections given above.

Lemma 3.2. A rotation $r_{\theta}$ about a line $l$ passing through $O=(0,0,0)$ is an isometry if and only if $r_{\theta} \in R_{c}=R_{1} \cup R_{2} \cup R_{3}$ such that

$R_{1}=\left\{r_{\theta} \mid \theta \in\{\pi / 2, \pi, 3 \pi / 2\}\right.$, rotation axis has a direction vector in $\left.D_{1}\right\}$

$R_{2}=\left\{r_{\theta} \mid \theta \in\{2 \pi / 3,4 \pi / 3\}\right.$, rotation axis has a direction vector in $\left.D_{2}\right\}$

$R_{3}=\left\{r_{\theta} \mid \theta=\pi\right.$, rotation axis has a direction vector in $\left.D_{3}\right\}$

where $D_{1}=\{(1,0,0),(0,1,0),(0,0,1)\}, D_{2}=\{(1,1,1),(-1,1,1),(1,-1,1),(1,1,-1)\}$ and $D_{3}=\{(1,1,0),(1,0,1),(0,1,1),(1,-1,0),(1,0,-1),(0,1,-1)\}$. 
Proof. Consider the Euclidean rotation $r_{\theta}$ about a line $l$ with the direction of a unit vector $(p, q, r), r_{\theta}: \mathbb{R}_{c} \rightarrow \mathbb{R}_{c}$ which has a matrix representation as follows:

$$
\left[\begin{array}{lcl}
\cos \theta+p^{2}(1-\cos \theta) & p q(1-\cos \theta)-r \sin \theta & \operatorname{pr}(1-\cos \theta)+q \sin \theta \\
p q(1-\cos \theta)+r \sin \theta & \cos \theta+q^{2}(1-\cos \theta) & q r(1-\cos \theta)-p \sin \theta \\
\operatorname{pr}(1-\cos \theta)-q \sin \theta & q r(1-\cos \theta)+p \sin \theta & \cos \theta+r^{2}(1-\cos \theta)
\end{array}\right]
$$

Since a rotation about an axis $l$ can be expressed as composition of two reflections by two planes intersecting along the line $l$, it is sufficient to consider the rotations about the lines with direction vectors $(1,0,0),(0,1,0),(0,0,1),(1,1,0),(1,0,1)$, $(0,1,1),(1,-1,0),(1,0,-1),(0,1,-1),(1,1,1),(-1,1,1),(1,-1,1)$ or $(1,1,-1)$ by Lemma 3.2 . In order to find isometric rotations in $\mathbb{R}_{c}^{3}$, it will be enough to determine the rotations which preserve the lengths of the edges of the $d_{c}$-unit sphere.

Now consider the vertices points $A_{1}=(1,0,0), A_{2}=\left(\frac{1}{\sqrt{2}}, \frac{1}{\sqrt{2}}, 0\right)$ and $A_{19}=\left(\frac{1}{2 \sqrt{2}-1}, \frac{1}{2 \sqrt{2}-1}, \frac{1}{2 \sqrt{2}-1}\right)$ of $d_{c}$-unit sphere. Rotating $A_{1}, A_{2}$ and $A_{19}$ by an angle $\theta$ about the line $l$, we get

$$
\begin{gathered}
r_{\theta}\left(A_{1}\right)=\left(\cos \theta+p^{2}(1-\cos \theta), p q(1-\cos \theta)+r \sin \theta, p r(1-\cos \theta)-q \sin \theta\right) \\
r_{\theta}\left(A_{2}\right)=\frac{1}{\sqrt{2}}\left(\left(p^{2}+p q\right)(1-\cos \theta)+\cos \theta-r \sin \theta,\left(p q+q^{2}\right)(1-\cos \theta)+\cos \theta+r \sin \theta\right. \\
\quad(p r+q r)(1-\cos \theta)+(p-q) \sin \theta) \\
r_{\theta}\left(A_{19}\right)=\frac{1}{2 \sqrt{2}-1}\left(\left(p^{2}+p q+p r\right)(1-\cos \theta)+\cos \theta+(q-r) \sin \theta\right. \\
\left(p q+q^{2}+q r\right)(1-\cos \theta)+\cos \theta+(r-p) \sin \theta \\
\left.\left(p r+q r+r^{2}\right)(1-\cos \theta)+\cos \theta+(p-q) \sin \theta\right) .
\end{gathered}
$$

Clearly $d_{c}\left(A_{1}, A_{2}\right)=2 \sqrt{2}-2$ and $d_{c}\left(A_{2}, A_{19}\right)=(9-3 \sqrt{2}) / 7$. If $r_{\theta}$ preserves $d_{c^{-}}$ distances, we must look for $\theta, \theta \neq 0$, which implies $d_{c}\left(r_{\theta}\left(A_{1}\right), r_{\theta}\left(A_{2}\right)\right)=2 \sqrt{2}-2$ and $d_{c}\left(r_{\theta}\left(A_{2}\right), r_{\theta}\left(A_{19}\right)\right)=(9-3 \sqrt{2}) / 7$. Thus,

$$
\begin{aligned}
d_{c}\left(r_{\theta}\left(A_{1}\right), r_{\theta}\left(A_{2}\right)\right)= & \max \left\{\left|\alpha_{1}\right|,\left|\beta_{1}\right|,\left|\gamma_{1}\right|\right\}+(\sqrt{2}-1) \\
& \min \left\{\left|\alpha_{1}\right|+\left|\beta_{1}\right|,\left|\alpha_{1}\right|+\left|\gamma_{1}\right|,\left|\beta_{1}\right|+\left|\gamma_{1}\right|\right\}=2 \sqrt{2}-2, \\
d_{c}\left(r_{\theta}\left(A_{2}\right), r_{\theta}\left(A_{19}\right)\right)=\max \left\{\left|\alpha_{2}\right|,\left|\beta_{2}\right|,\left|\gamma_{2}\right|\right\}+(\sqrt{2}-1) & \min \left\{\left|\alpha_{2}\right|+\left|\beta_{2}\right|,\left|\alpha_{2}\right|+\left|\gamma_{2}\right|,\left|\beta_{2}\right|+\left|\gamma_{2}\right|\right\}=(9-3 \sqrt{2}) / 7,
\end{aligned}
$$

where

$$
\begin{aligned}
& \text { where } \\
& \begin{aligned}
& \alpha_{1}=\frac{1}{\sqrt{2}}\left[\left((\sqrt{2}-1) p^{2}-p q\right)(1-\cos \theta)+(\sqrt{2}-1) \cos \theta+r \sin \theta\right], \\
& \beta_{1}=\frac{1}{\sqrt{2}}\left[\left((\sqrt{2}-1) p q-q^{2}\right)(1-\cos \theta)+(\sqrt{2}-1) r \sin \theta+\cos \theta\right], \\
& \gamma_{1}=\frac{1}{\sqrt{2}}[((\sqrt{2}-1) p r-q r)(1-\cos \theta)-((\sqrt{2}-1) q+p) \sin \theta]
\end{aligned}
\end{aligned}
$$

and

$$
\begin{aligned}
& \alpha_{2}=\frac{1}{4-\sqrt{2}}\left(\left[(1-\sqrt{2})\left(p^{2}+p q\right)+\sqrt{2} p r\right](1-\cos \theta)+(1-\sqrt{2}) \cos \theta+(\sqrt{2} q+(\sqrt{2}-1) r) \sin \theta\right), \\
& \beta_{2}=\frac{1}{4-\sqrt{2}}\left(\left[(1-\sqrt{2})\left(p q+q^{2}\right)+\sqrt{2} q r\right](1-\cos \theta)+(1-\sqrt{2}) \cos \theta-(\sqrt{2} p+(\sqrt{2}-1) r) \sin \theta\right), \\
& \gamma_{2}=\frac{1}{4-\sqrt{2}}\left(\left[(1-\sqrt{2})(p r+q r)+\sqrt{2} r^{2}\right](1-\cos \theta)+\sqrt{2} \cos \theta-(\sqrt{2}-1)(p-q) \sin \theta\right) .
\end{aligned}
$$

If direction vector of $l$ is in $D_{1}$, say $(1,0,0)$, then $(p, q, r)=(1,0,0)$. Using these values of $p, q, r$ in the equations $d_{c}\left(r_{\theta}\left(A_{1}\right), r_{\theta}\left(A_{2}\right)\right)=2 \sqrt{2}-2$ and 
$d_{c}\left(r_{\theta}\left(A_{2}\right), r_{\theta}\left(A_{19}\right)\right)=(9-3 \sqrt{2}) / 7$ one obtains

$$
\begin{gathered}
\max \{|\sqrt{2}-1|,|\cos \theta|,|\sin \theta|\}+(\sqrt{2}-1) \\
\min \{|\sqrt{2}-1|+|\cos \theta|,|\cos \theta|+|\sin \theta|,|\sqrt{2}-1|+|\sin \theta|\}=4-2 \sqrt{2} \\
\max \{\sqrt{2}-1,|(1-\sqrt{2}) \cos \theta-\sqrt{2} \sin \theta|,|\sqrt{2} \cos \theta+(1-\sqrt{2}) \sin \theta|\}+(\sqrt{2}-1) \\
\min \{\sqrt{2}-1+|(1-\sqrt{2}) \cos \theta-\sqrt{2} \sin \theta|, \sqrt{2}-1+|\sqrt{2} \cos \theta+(1-\sqrt{2}) \sin \theta|, \\
|(1-\sqrt{2}) \cos \theta-\sqrt{2} \sin \theta|+|\sqrt{2} \cos \theta+(1-\sqrt{2}) \sin \theta|\}=6-3 \sqrt{2} .
\end{gathered}
$$

From this equation, one gets $\theta=\pi / 2, \pi$ or $3 \pi / 2$. That is, every Euclidean rotation about $x$-axis with $\theta=\pi / 2, \pi$ or $3 \pi / 2$ is an isometry of $\mathbb{R}_{c}^{3}$. Similarly, if direction vector of $l$ is one of $(0,1,0)$ or $(0,0,1)$, then $\theta=\pi / 2, \pi$ or $3 \pi / 2$.

If direction vector of $l$ is in $D_{2}$, say $(1,1,1)$, then $(p, q, r)=(1 / \sqrt{3}, 1 / \sqrt{3}, 1 / \sqrt{3})$. Using these values of $p, q, r$ in the equations $d_{c}\left(r_{\theta}\left(A_{1}\right), r_{\theta}\left(A_{2}\right)\right)=2 \sqrt{2}-2$ and $d_{c}\left(r_{\theta}\left(A_{2}\right), r_{\theta}\left(A_{19}\right)\right)=(9-3 \sqrt{2}) / 7$. One gets

$$
\begin{aligned}
d_{c}\left(r_{\theta}\left(A_{1}\right), r_{\theta}\left(A_{2}\right)\right)= & \frac{1}{3 \sqrt{2}}\left(\max \left\{\left|\alpha_{3}\right|,\left|\beta_{3}\right|,\left|\gamma_{3}\right|\right\}+(\sqrt{2}-1)\right. \\
d_{c}\left(r_{\theta}\left(A_{2}\right), r_{\theta}\left(A_{19}\right)\right)= & \frac{1}{4-\sqrt{2}}\left(\max \left\{\left|\alpha_{3}\right|+\left|\beta_{3}\right|,\left|\alpha_{3}\right|+\left|\gamma_{3}\right|,\left|\beta_{4}\right|,\left|\gamma_{4}\right|\right\}+(\sqrt{2}-1)\right. \\
& \left.\quad \min \left\{\left|\alpha_{3}\right|+\left|\beta_{4}\right|,\left|\alpha_{4}\right|+\left|\gamma_{4}\right|,\left|\beta_{4}\right|+\left|\gamma_{4}\right|\right\}\right)=(9-3 \sqrt{2}) / 7
\end{aligned}
$$

where

$$
\begin{aligned}
& \text { where } \\
& \begin{aligned}
\alpha_{3} & =(\sqrt{2}-2)+(2 \sqrt{2}-1) \cos \theta+\sqrt{3} \sin \theta \\
\beta_{3} & =(\sqrt{2}-2)+(-\sqrt{2}-1) \cos \theta+(\sqrt{2}-1) \sqrt{3} \sin \theta \\
\gamma_{3} & =(\sqrt{2}-2)(1-\cos \theta)-\sqrt{6} \sin \theta
\end{aligned}
\end{aligned}
$$

and

$$
\begin{aligned}
\alpha_{4} & =\frac{2-\sqrt{2}}{3}(1-\cos \theta)+(1-\sqrt{2}) \cos \theta+\frac{2 \sqrt{2}-1}{\sqrt{3}} \sin \theta \\
\beta_{4} & =\frac{2-\sqrt{2}}{3}(1-\cos \theta)+(1-\sqrt{2}) \cos \theta-\frac{2 \sqrt{2}-1}{\sqrt{3}} \sin \theta \\
\gamma_{4} & =\frac{2-\sqrt{2}}{3}(1-\cos \theta)+\sqrt{2} \cos \theta .
\end{aligned}
$$

From this equation, one obtains $\theta=2 \pi / 3$ or $4 \pi / 3$. That is, every Euclidean rotation about the line $l$ that has the direction vector $(1,1,1)$ with $\theta=2 \pi / 3$ or $4 \pi / 3$ is an isometry of $\mathbb{R}_{c}^{3}$. Similarly, if direction vector of $l$ is one of $(-1,1,1),(1,-1,1)$, or $(1,1,-1)$, then $\theta=2 \pi / 3$, or $4 \pi / 3$.

If direction vector of $l$ is in $D_{3}$, say $(1,1,0)$, then $(p, q, r)=(1 / \sqrt{2}, 1 / \sqrt{2}, 0)$. Using these values of $p, q, r$ in the equations $d_{c}\left(r_{\theta}\left(A_{1}\right), r_{\theta}\left(A_{2}\right)\right)=2 \sqrt{2}-2$ and $d_{c}\left(r_{\theta}\left(A_{2}\right), r_{\theta}\left(A_{19}\right)\right)=(9-3 \sqrt{2}) / 7$ one gets

$$
\begin{array}{r}
\frac{1}{2}(\max \{|\cos \theta+(1-\sqrt{2})|,|(1-\sqrt{2})-\cos \theta|, \sqrt{2}|\sin \theta|\}+(\sqrt{2}-1) \\
\min \{|\cos \theta+(1-\sqrt{2})|+|(1-\sqrt{2})-\cos \theta|,|(1-\sqrt{2})-\cos \theta|+\sqrt{2}|\sin \theta|, \\
|(1-\sqrt{2})-\cos \theta|+\sqrt{2}|\sin \theta|\})=2 \sqrt{2}-2
\end{array}
$$


and

$$
\begin{array}{r}
\frac{1}{4-\sqrt{2}}(\max \{|1-\sqrt{2}+\sin \theta|,|1-\sqrt{2}-\sin \theta|,|\sqrt{2} \cos \theta|\}+(\sqrt{2}-1) \\
\min \{|1-\sqrt{2}+\sin \theta|+|1-\sqrt{2}-\sin \theta|,|1-\sqrt{2}+\sin \theta|+|\sqrt{2} \cos \theta| \\
|1-\sqrt{2}-\sin \theta|+|\sqrt{2} \cos \theta|\})=(9-3 \sqrt{2}) / 7
\end{array}
$$

From this equation, one gets $\theta=\pi$. That is, every Euclidean rotation about the line $l$ that has the direction vector $(1,1,0)$ with $\theta=\pi$ is an isometry of $\mathbb{R}_{c}^{3}$. Similarly, if direction vector of $l$ is one of $(1,0,1),(0,1,1),(1,-1,0),(1,0,-1)$ or $(0,1,-1)$, then $\theta=\pi$.

Conversely, if $r_{\theta}(X)=Y$, then it can easily be check that $\mu_{O X}=\mu_{O Y}$ for all possible cases as in Lemma 3.2. For instance:

\begin{tabular}{c|c|c|c|c} 
rotation & $(1,0,0)$ & $\frac{1}{\sqrt{2}}(1,1,0)$ & $\frac{1}{\sqrt{3}}(1,1,1)$ & $\ldots$ \\
& $\theta=\pi / 2$ & $\theta=\pi$ & $\theta=2 \pi / 3$ & \\
\hline$\left(p_{2}, q_{2}, r_{2}\right)$ & $\left(p_{1},-r_{1}, q_{1}\right)$ & $\left(q_{1}, p_{1},-r_{1}\right)$ & $\left(r_{1}, p_{1}, q_{1}\right)$ & $\cdots$
\end{tabular}

Thus, the set $R_{c}$ of isometric rotations about the lines passing through the origin consists of exactly twenty-three Euclidean rotations mentioned above.

Clearly the inversion $\sigma_{O}$ about $O=(0,0,0)$, which maps $(x, y, z)$ to $(-x,-y,-z)$, is an isometry of $\mathbb{R}_{c}$ and preserves the $d_{c}$-unit sphere. $\sigma_{O}$ will be useful to give the following two lemmas:

Lemma 3.3. There are only six rotary reflections about $O$ that preserve the $d_{c^{-}}$ distances.

Proof. Since a rotary reflection $\rho:=\sigma_{\Pi} \sigma_{\Delta} \sigma_{\Gamma}=\sigma_{\Pi} r_{\theta}, r_{\theta} \in R_{c}, \Gamma$ and $\Delta$ perpendicular to $\Pi$, one has to consider both the possible 9 cases for $\Pi$ and 13 choices for the axes of rotations by Lemma 3.2 and Lemma 3.3. But notice that non of the lines in $D_{2}$ is perpendicular to any one of planes given in Lemma 3.2.

If $\Pi$ stands for the plane $x=0$, then $(1,0,0)$ is unit direction vector of $r_{\theta}$ and $\rho(x, y, z)=\sigma_{\Pi} r_{\theta}(x, y, z)=(-x, y \cos \theta-z \sin \theta, y \sin \theta+z \cos \theta)$. Consequently, $\rho\left(A_{1}\right)=$ $(-1,0,0), \rho\left(A_{2}\right)=(-1 / \sqrt{2}, \cos \theta / \sqrt{2}, \sin \theta / \sqrt{2})$ and $\rho\left(A_{19}\right)=\left(\frac{-1}{2 \sqrt{2}-1}, \frac{\cos \theta-\sin \theta}{2 \sqrt{2}-1}, \frac{\cos \theta+\sin \theta}{2 \sqrt{2}-1}\right)$.

Since $d_{c}\left(A_{1}, A_{2}\right)=2 \sqrt{2}-2$ and $d_{c}\left(A_{2}, A_{9}\right)=(9-3 \sqrt{2}) / 7$,

$$
\begin{aligned}
& d_{c}\left(\rho\left(A_{1}\right), \rho\left(A_{2}\right)\right)= 2 \sqrt{2}-2 \Leftrightarrow \max \{|\sqrt{2}-1|,|\cos \theta|,|\sin \theta|\}+(\sqrt{2}-1) \\
& \min \{|\sqrt{2}-1|+|\cos \theta|,|\sqrt{2}-1|+|\sin \theta|,|\cos \theta|+|\sin \theta|\}=4-2 \sqrt{2} \Leftrightarrow \theta \in\{\pi / 2, \pi, 3 \pi / 2\} \\
& d_{c}\left(\rho\left(A_{2}\right), \rho\left(A_{19}\right)\right)=(9-3 \sqrt{2}) / 7 \Leftrightarrow \\
& \max \{\sqrt{2}-1,|(\sqrt{2}-1) \cos \theta+\sqrt{2} \sin \theta|,|(\sqrt{2}-1) \sin \theta-\sqrt{2} \cos \theta|\} \\
&+(\sqrt{2}-1) \min \{\sqrt{2}-1+|(\sqrt{2}-1) \cos \theta+\sqrt{2} \sin \theta|, \sqrt{2}-1+|(\sqrt{2}-1) \sin \theta-\sqrt{2} \cos \theta| \\
&|(\sqrt{2}-1) \cos \theta+\sqrt{2} \sin \theta|+|(\sqrt{2}-1) \sin \theta-\sqrt{2} \cos \theta|\}=6-3 \sqrt{2} \\
& \Leftrightarrow \theta \in\{\pi / 2, \pi, 3 \pi / 2\} .
\end{aligned}
$$

Now it is easy to show that $\sigma_{\Pi} r_{\pi}=\sigma_{O}$, and therefore there are exactly two rotary reflections obtained using the plane $x=0$. Similarly, one can easily obtain new rotary reflections using the planes $y=0$ and $z=0$ as $\Pi$. 
If $\Pi$ denotes the plane $x+y=0$, then $(1 / \sqrt{2}, 1 / \sqrt{2}, 0)$ is unit direction vector of $r_{\theta}$ and

$$
\begin{aligned}
& \rho(x, y, z)= \sigma_{\Pi} r_{\theta}(x, y, z) \\
&=\left(\left(\frac{\cos \theta-1}{2}\right) x-\left(\frac{1+\cos \theta}{2}\right) y+\frac{\sin \theta}{\sqrt{2}} z,\right. \\
&\left(\frac{-1-\cos \theta}{2}\right) x+\left(\frac{\cos \theta-1}{2}\right) y-\frac{\sin \theta}{\sqrt{2}} z, \\
&\left.\frac{-\sin \theta}{\sqrt{2}} x+\frac{\sin \theta}{\sqrt{2}} y+\cos \theta z\right) .
\end{aligned}
$$

Clearly $\rho\left(A_{1}\right)=\left(\frac{1}{2}(\cos \theta-1), \frac{-1}{2}(1+\cos \theta), \frac{-1}{\sqrt{2}} \sin \theta\right), \rho\left(A_{2}\right)=\left(\frac{-1}{\sqrt{2}}, \frac{-1}{\sqrt{2}}, 0\right)$,

$$
\begin{aligned}
& \rho\left(A_{19}\right)=\frac{1}{2 \sqrt{2}-1}\left(-1+\frac{\sin \theta}{\sqrt{2}},-1-\frac{\sin \theta}{\sqrt{2}}, \cos \theta\right) \text { and } \\
& d_{c}\left(\rho\left(A_{1}\right), \rho\left(A_{2}\right)\right)=2 \sqrt{2}-2 \Leftrightarrow \\
& \max \{|\cos \theta+\sqrt{2}-1|,|-\cos \theta+\sqrt{2}-1|, \sqrt{2}|\sin \theta|\}+(\sqrt{2}-1) \\
& \min \{|\cos \theta+\sqrt{2}-1|+|-\cos \theta+\sqrt{2}-1|,|\cos \theta+\sqrt{2}-1|+\sqrt{2}|\sin \theta|, \\
& |-\cos \theta+\sqrt{2}-1|+\sqrt{2}|\sin \theta|\}=4 \sqrt{2}-4 \\
& \Leftrightarrow \theta=\pi \\
& d_{c}\left(\rho\left(A_{2}\right), \rho\left(A_{19}\right)\right)=(9-3 \sqrt{2}) / 7 \Leftrightarrow \\
& \max \{|1-\sqrt{2}-\sin \theta|,|1-\sqrt{2}-\sin \theta|,|\sqrt{2} \cos \theta|\}+(\sqrt{2}-1) \\
& \min \{|1-\sqrt{2}-\sin \theta|+|1-\sqrt{2}-\sin \theta|,|1-\sqrt{2}-\sin \theta|+|\sqrt{2} \cos \theta|, \\
& |1-\sqrt{2}-\sin \theta|+|\sqrt{2} \cos \theta|\}=6-3 \sqrt{2} \\
& \Leftrightarrow \theta=\pi
\end{aligned}
$$

but $\rho=\sigma_{\Pi} r_{\pi}=\sigma_{O}$ the inversion about $O=(0,0,0)$. That is, there is no new rotary reflection in this case.

Similarly, it is easily seen that there is no new rotary reflection if $\Pi$ is any of the remaining planes $x-y=0, x+z=0, x-z=0, y+z=0, y-z=0$ and rotations axes are parallel to any one of $(1,-1,0),(1,0,1),(1,0,-1),(0,1,1),(0,1,-1)$, respectively.

Lemma 3.4. There exist only eight rotary inversions about $O$ that preserves the $d_{c}$-distances.

Proof. Since a rotary inversion $\rho:=\sigma_{O} \sigma_{\Delta} \sigma_{\Gamma}=\sigma_{O} r_{\theta}, r_{\theta} \in R_{c}$, one has to consider possible 13 cases for the axes of rotations by Lemma 3.3.

If $r_{\theta}$ denotes the rotations about $x$-axis, then $(1,0,0)$ is unit direction vector of $r_{\theta}$ and $\rho(x, y, z)=\sigma_{O} r_{\theta}(x, y, z)=(-x,-y \cos \theta+z \sin \theta,-y \sin \theta-z \cos \theta)$. Consequently, $\rho\left(A_{1}\right)=(-1,0,0), \rho\left(A_{2}\right)=(-1 / \sqrt{2},-\cos \theta / \sqrt{2},-\sin \theta / \sqrt{2})$ and $\rho\left(A_{19}\right)=\frac{1}{2 \sqrt{2}-1}(-1,-\cos \theta+\sin \theta,-\cos \theta-\sin \theta)$. Since $d_{c}\left(A_{1}, A_{2}\right)=2 \sqrt{2}-2$ and 


$$
\begin{gathered}
d_{c}\left(A_{2}, A_{9}\right)=(9-3 \sqrt{2}) / 7, \\
\begin{array}{r}
d_{c}\left(\rho\left(A_{1}\right), \rho\left(A_{2}\right)\right)=2 \sqrt{2}-2 \Leftrightarrow \\
\max \{|\sqrt{2}-1|,|\cos \theta|,|\sin \theta|\}+(\sqrt{2}-1) \\
\min \{|\sqrt{2}-1|+|\cos \theta|,|\sqrt{2}-1|+|\sin \theta|,|\cos \theta|+|\sin \theta|\} \\
=4-2 \sqrt{2} \Leftrightarrow \theta \in\{\pi / 2, \pi, 3 \pi / 2\} . \\
d_{c}\left(\rho\left(A_{2}\right), \rho\left(A_{19}\right)\right)=\frac{9-3 \sqrt{2}}{7} \Leftrightarrow \\
\max \{\sqrt{2}-1,|(1-\sqrt{2}) \cos \theta-\sqrt{2} \sin \theta|,|(1-\sqrt{2}) \sin \theta+\sqrt{2} \cos \theta|\}+(\sqrt{2}-1) \\
\min \{\sqrt{2}-1+|(1-\sqrt{2}) \cos \theta-\sqrt{2} \sin \theta|, \sqrt{2}-1+|(1-\sqrt{2}) \sin \theta+\sqrt{2} \cos \theta| \\
|(1-\sqrt{2}) \cos \theta-\sqrt{2} \sin \theta|+|(1-\sqrt{2}) \sin \theta+\sqrt{2} \cos \theta|\} \\
=6-3 \sqrt{2} \Leftrightarrow \theta \in\{\pi / 2, \pi, 3 \pi / 2\} .
\end{array}
\end{gathered}
$$

Now it is easy to show that $\sigma_{O} r_{\theta}$ is a rotary reflection or reflection, and therefore there is no new rotary inversion in this case. Similarly, one can easily see that there is no new rotary inversion using the rotations about $y, z$-axis.

If $r_{\theta}$ stands for rotation about $l$ parallel to $(1,1,0)$, then $(1 / \sqrt{2}, 1 / \sqrt{2}, 0)$ is unit direction vector of $r_{\theta}$ and

$$
\begin{aligned}
\rho(x, y, z)= & \sigma_{O} r_{\theta}(x, y, z) \\
= & \left(\left(\frac{-1-\cos \theta}{2}\right) x+\left(\frac{\cos \theta-1}{2}\right) y-\frac{\sin \theta}{\sqrt{2}} z,\right. \\
& \left(\frac{\cos \theta-1}{2}\right) x+\left(\frac{-1-\cos \theta}{2}\right) y+\frac{\sin \theta}{\sqrt{2}} z, \\
& \left.\frac{\sin \theta}{\sqrt{2}} x-\frac{\sin \theta}{\sqrt{2}} y-\cos \theta z\right) .
\end{aligned}
$$

Obviously $\rho\left(A_{1}\right)=\left(\frac{-1}{2}(1+\cos \theta), \frac{1}{2}(\cos \theta-1), \frac{1}{\sqrt{2}} \sin \theta\right), \rho\left(A_{2}\right)=\left(\frac{-1}{\sqrt{2}}, \frac{-1}{\sqrt{2}}, 0\right)$, $\rho\left(A_{19}\right)=\frac{1}{2 \sqrt{2}-1}\left(-1-\frac{\sin \theta}{\sqrt{2}},-1+\frac{\sin \theta}{\sqrt{2}},-\cos \theta\right)$ and

$$
\begin{gathered}
d_{c}\left(\rho\left(A_{1}\right), \rho\left(A_{2}\right)\right)=2 \sqrt{2}-2 \Leftrightarrow \\
\max \{|\cos \theta+\sqrt{2}-1|,|-\cos \theta+\sqrt{2}-1|, \sqrt{2}|\sin \theta|\}+(\sqrt{2}-1) \\
\min \{|\cos \theta+\sqrt{2}-1|+|-\cos \theta+\sqrt{2}-1|,|\cos \theta+\sqrt{2}-1|+\sqrt{2}|\sin \theta|, \\
|-\cos \theta+\sqrt{2}-1|+\sqrt{2}|\sin \theta|\}=4 \sqrt{2}-4 \Leftrightarrow \theta=\pi \\
d_{c}\left(\rho\left(A_{2}\right), \rho\left(A_{19}\right)\right)=(9-3 \sqrt{2}) / 7 \Leftrightarrow \\
\max \{|1-\sqrt{2}+\sin \theta|,|1-\sqrt{2}-\sin \theta|,|\sqrt{2} \cos \theta|\}+(\sqrt{2}-1) \\
\min \{|1-\sqrt{2}+\sin \theta|+|1-\sqrt{2}-\sin \theta|,|1-\sqrt{2}+\sin \theta|+|\sqrt{2} \cos \theta|, \\
|1-\sqrt{2}-\sin \theta|+|\sqrt{2} \cos \theta|\}=6-3 \sqrt{2} \Leftrightarrow \theta=\pi
\end{gathered}
$$

but $\rho=\sigma_{O} r_{\pi}$ is a rotary reflection or reflection. That is, there is no new rotary inversion in this case.

Similarly, it is easily see that there is no new rotary inversion if $r_{\theta}$ any of the remaining rotation axes parallel to $(1,-1,0),(1,0,1),(1,0,-1),(0,1,1),(0,1,-1)$. 
If $r_{\theta}$ denotes rotation about an axis parallel to $(1,1,1)$, then $(1 / \sqrt{3}, 1 / \sqrt{3}, 1 / \sqrt{3})$ is unit direction vector of $r_{\theta}$ and

$$
\begin{aligned}
\rho(x, y, z)= & \sigma_{O} r_{\theta}(x, y, z) \\
= & \left(\left(\frac{-2 \cos \theta-1}{3}\right) x+\left(\frac{\cos \theta+\sqrt{3} \sin \theta-1}{3}\right) y+\left(\frac{\cos \theta-\sqrt{3} \sin \theta-1}{3}\right) z,\right. \\
& \quad\left(\frac{\cos \theta-\sqrt{3} \sin \theta-1}{3}\right) x+\left(\frac{-2 \cos \theta-1}{3}\right) y+\left(\frac{\cos \theta+\sqrt{3} \sin \theta-1}{3}\right) z, \\
& \left.\left(\frac{\cos \theta+\sqrt{3} \sin \theta-1}{3}\right) x+\left(\frac{\cos \theta-\sqrt{3} \sin \theta-1}{3}\right) y+\left(\frac{-2 \cos \theta-1}{3}\right) z\right) .
\end{aligned}
$$

Clearly $\rho\left(A_{1}\right)=\left(\frac{-2 \cos \theta-1}{3}, \frac{\cos \theta-\sqrt{3} \sin \theta-1}{3}, \frac{\cos \theta+\sqrt{3} \sin \theta-1}{3}\right)$,

$$
\begin{gathered}
\rho\left(A_{2}\right)=\left(\frac{-2-\cos \theta+\sqrt{3} \sin \theta}{3 \sqrt{2}}, \frac{-2-\cos \theta-\sqrt{3} \sin \theta}{3 \sqrt{2}}, \frac{2 \cos \theta-2}{3 \sqrt{2}}\right), \\
\rho\left(A_{19}\right)=\left(\frac{-1}{2 \sqrt{2}-1}, \frac{-1}{2 \sqrt{2}-1}, \frac{-1}{2 \sqrt{2}-1}\right) \text { and } \\
d_{c}\left(\rho\left(A_{1}\right), \rho\left(A_{2}\right)\right)=2 \sqrt{2}-2 \Leftrightarrow \max \{|-2+\sqrt{2}+(2 \sqrt{2}-1) \cos \theta+\sqrt{3} \sin \theta|, \\
|(-1-\sqrt{2}) \cos \theta+(-\sqrt{2}+\sqrt{3} \sin \theta)(\sqrt{2}-1)|,|(2-\sqrt{2})(\cos \theta-1)-\sqrt{3} \sin \theta|\}+(\sqrt{2}-1) \\
\min \{|-2+\sqrt{2}+(2 \sqrt{2}-1) \cos \theta+\sqrt{3} \sin \theta|+|(-1-\sqrt{2}) \cos \theta+(-\sqrt{2}+\sqrt{3} \sin \theta)(\sqrt{2}-1)|, \\
|-2+\sqrt{2}+(2 \sqrt{2}-1) \cos \theta+\sqrt{3} \sin \theta|+|(2-\sqrt{2})(\cos \theta-1)-\sqrt{3} \sin \theta|, \\
|(-1-\sqrt{2}) \cos \theta+(-\sqrt{2}+\sqrt{3} \sin \theta)(\sqrt{2}-1)|+|(2-\sqrt{2})(\cos \theta-1)-\sqrt{3} \sin \theta|\} \\
d_{c}\left(\rho\left(A_{2}\right), \rho\left(A_{19}\right)\right)=(9-3 \sqrt{2}) / 7 \Leftrightarrow \max \{\mid 2-\sqrt{2}+(2 \sqrt{2}-1)(-\cos \theta+\sqrt{3} \sin \theta \mid, \\
\mid 2-\sqrt{2}+(2 \sqrt{2}-1)(-\cos \theta-\sqrt{3} \sin \theta|,| 2-\sqrt{2}+2(2 \sqrt{2}-1) \cos \theta \mid\}+(\sqrt{2}-1) \\
\min \{\mid 2-\sqrt{2}+(2 \sqrt{2}-1)(-\cos \theta+\sqrt{3} \sin \theta|+| 2-\sqrt{2}+(2 \sqrt{2}-1)(-\cos \theta-\sqrt{3} \sin \theta \mid \\
\mid 2-\sqrt{2}+(2 \sqrt{2}-1)(-\cos \theta+\sqrt{3} \sin \theta|+| 2-\sqrt{2}+2(2 \sqrt{2}-1) \cos \theta \mid, \\
\mid 2-\sqrt{2}+(2 \sqrt{2}-1)(-\cos \theta-\sqrt{3} \sin \theta|+| 2-\sqrt{2}+2(2 \sqrt{2}-1) \cos \theta \mid\}=18-9 \sqrt{2} \\
\Leftrightarrow \theta \in\{2 \pi / 3,4 \pi / 3\} .
\end{gathered}
$$

Therefore there exactly two rotary inversions obtained using rotation about axis parallel to $(1,1,1)$.

Similarly, it is easily obtain that there are two new rotary inversion each of the remaining rotation axes parallel to $(-1,1,1),(1,-1,1),(1,1,-1)$. That is, there are eight rotary inversions that preserve $d_{c}$-distance.

It can be easily check that $\sigma_{O} \sigma_{\Delta}=r_{\pi}, r_{\pi} \in R_{1} \cup R_{3}$.

Thus we have the deltoidal icositetrahedron group, $G(D)$, consisting of nine reflections about planes, twenty-three rotations, six rotary reflections, eight rotary inversions, one inversion and the identity. That is, the Euclidean symmetry group of the deltoidal isocitetrahedron. Deltoidal icositetrahedron group $G(D)$ is isomorph to the cube group $S(C)$ which is the (Euclidean) symmetry group of the cube.

Now, let us show that all isometries of $\mathbb{R}_{c}^{3}$ are in $T(3) . G(D)$. 
Definition 3.1. Let $A=\left(a_{1}, a_{2}, a_{3}\right), B=\left(b_{1}, b_{2}, b_{3}\right)$ be two points in $\mathbb{R}_{c}^{3}$. The minimum distance set of $A, B$ is defined by

$$
\left\{X \mid d_{c}(A, X)+d_{c}(B, X)=d_{c}(A, B)\right\}
$$

and denoted by $[A B]$.

In general, $[A B]$ represent a parallelepiped with diagonal $A B$ as in Fig. 3 .

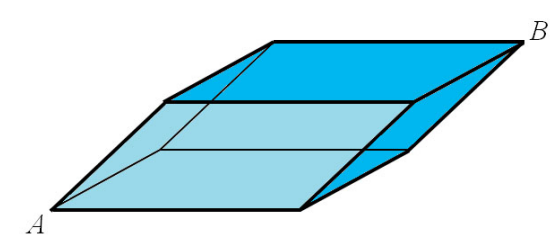

Figure 3

In particular, if $A B$ is parallel to any of the coordinate axes or $A B$ is on a plane which is the parallel to any coordinate plane and slope of $A B$ is \pm 1 , then $[A B]=A B$.

Proposition 3.2. Let $\phi: \mathbb{R}_{c}^{3} \rightarrow \mathbb{R}_{c}^{3}$ be an isometry and let $[A B]$ be the parallelepiped. Then

$$
\phi([A B])=[\phi(A) \phi(B)]
$$

Proof. Let $Y \in \phi([A B])$. Then,

$$
\begin{aligned}
Y \in \phi([A B]) & \Leftrightarrow \exists X \in[A B] \ni Y=\phi(X) \\
& \Leftrightarrow d_{c}(A, X)+d_{c}(X, B)=d_{c}(A, B) \\
& \Leftrightarrow d_{c}(\phi(A), \phi(X))+d_{c}(\phi(X), \phi(B))=d_{c}(\phi(A), \phi(B)) \\
& \Leftrightarrow Y=\phi(X) \in[\phi(A) \phi(B)] .
\end{aligned}
$$

Corollary 3.1. Let $\phi: \mathbb{R}_{c}^{3} \rightarrow \mathbb{R}_{c}^{3}$ be an isometry and let $[A B]$ be a parallelepiped. Then $\phi$ maps vertices to vertices and preserves the lengths of edges of $[A B]$.

Proposition 3.3. Let $f: \mathbb{R}_{c}^{3} \rightarrow \mathbb{R}_{c}^{3}$ be an isometry such that $f(O)=O$. Then $f$ is in $G(D)$.

Proof. Let $A_{1}=(1,0,0), A_{2}=\left(\frac{1}{\sqrt{2}}, \frac{1}{\sqrt{2}}, 0\right), A_{19}=\left(\frac{1}{2 \sqrt{2}-1}, \frac{1}{2 \sqrt{2}-1}, \frac{1}{2 \sqrt{2}-1}\right)$ and $D=\left(\frac{2 \sqrt{2}+3}{4-\sqrt{2}}, \frac{3 \sqrt{2}-1}{4-\sqrt{2}}, \frac{\sqrt{2}}{4-\sqrt{2}}\right)$. Consider $[O D]$ which is the parallelepiped with diagonal $O D$. 


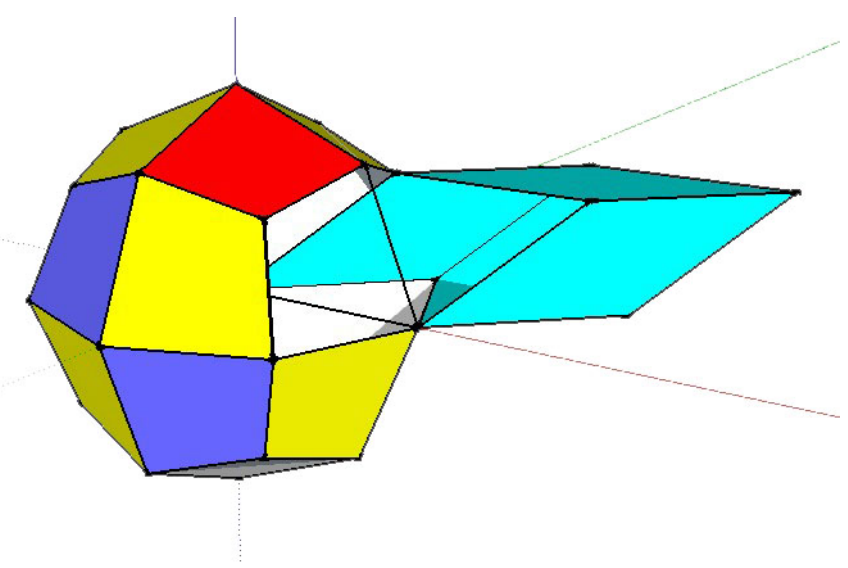

Figure 4

It is clear from the Fig. 4 that $f\left(A_{k}\right) \in A_{i} A_{j}, i \neq j \neq k, i, j, k \in\{1,2, \ldots, 26\}$. Since $f$ is an isometry by Corollary 3.8, $f\left(A_{1}\right), f\left(A_{2}\right)$ and $f\left(A_{19}\right)$ must be the vertices of the parallelepiped with diagonal $O D$. Therefore, if $f\left(A_{1}\right)=A_{i}$, then $i \in$ $\{1,3,5,7,17,18\}$. Similarly if $f\left(A_{2}\right)=A_{j}$ and $f\left(A_{19}\right)=A_{k}$, then $j \in\{2,4,6,8,9,10,11,12,13,14,15,16\}$ and $k \in\{19,20,21,22,23,24,25,26\}$. Here the points $f\left(A_{1}\right), f\left(A_{2}\right)$ and $f\left(A_{19}\right)$ are not elements of the line with direction vector $D_{1}, D_{2}, D_{3}$ which is given in Lemma 3.3. Also these points are on the same octant. Now the following six cases are possible:

1. If $f\left(A_{1}\right)=A_{1}$, then $f\left(A_{2}\right) \in\left\{A_{2}, A_{8}, A_{9}, A_{13}\right\}$ and $f\left(A_{19}\right) \in\left\{A_{19}, A_{22}, A_{23}, A_{26}\right\}$.

2. If $f\left(A_{1}\right)=A_{3}$, then $f\left(A_{2}\right) \in\left\{A_{2}, A_{4}, A_{10}, A_{14}\right\}$ and $f\left(A_{19}\right) \in\left\{A_{19}, A_{20}, A_{23}, A_{24}\right\}$.

3. If $f\left(A_{1}\right)=A_{5}$, then $f\left(A_{2}\right) \in\left\{A_{4}, A_{6}, A_{11}, A_{15}\right\}$ and $f\left(A_{19}\right) \in\left\{A_{20}, A_{21}, A_{24}, A_{25}\right\}$.

4. If $f\left(A_{1}\right)=A_{7}$, then $f\left(A_{2}\right) \in\left\{A_{6}, A_{8}, A_{12}, A_{16}\right\}$ and $f\left(A_{19}\right) \in\left\{A_{21}, A_{22}, A_{25}, A_{26}\right\}$.

5. If $f\left(A_{1}\right)=A_{17}$, then $f\left(A_{2}\right) \in\left\{A_{9}, A_{10}, A_{11}, A_{12}\right\}$ and $f\left(A_{19}\right) \in\left\{A_{19}, A_{20}, A_{21}, A_{22}\right\}$.

6. If $f\left(A_{1}\right)=A_{18}$, then $f\left(A_{2}\right) \in\left\{A_{13}, A_{14}, A_{15}, A_{16}\right\}$ and $f\left(A_{19}\right) \in\left\{A_{23}, A_{24}, A_{25}, A_{26}\right\}$.

In each case it is easy to show that $f$ is unique and is in $G(D)$. For instance in the first case:

If $f\left(A_{2}\right)=A_{2}$ and $f\left(A_{19}\right)=A_{19}$, then $f$ is the identity.

If $f\left(A_{2}\right)=A_{2}$ and $f\left(A_{19}\right)=A_{23}$, then $f=\sigma_{\Delta}$ such that $\Delta: z=0$.

If $f\left(A_{2}\right)=A_{8}$ and $f\left(A_{19}\right)=A_{22}$, then $f=\sigma_{\Delta}$ such that $\Delta: y=0$.

If $f\left(A_{2}\right)=A_{8}$ and $f\left(A_{19}\right)=A_{26}$, then $f=r_{\pi}$ with rotation axis $\|(1,0,0)$.

If $f\left(A_{2}\right)=A_{9}$ and $f\left(A_{19}\right)=A_{19}$, then $f=\sigma_{\Delta}$ such that $\Delta: y-z=0$.

If $f\left(A_{2}\right)=A_{9}$ and $f\left(A_{19}\right)=A_{22}$, then $f=r_{\pi / 2}$ with rotation axis $\|(1,0,0)$.

If $f\left(A_{2}\right)=A_{13}$ and $f\left(A_{19}\right)=A_{23}$, then $f=r_{3 \pi / 2}$ with rotation axis $\|(1,0,0)$.

If $f\left(A_{2}\right)=A_{13}$ and $f\left(A_{19}\right)=A_{26}$, then $f=\sigma_{\Delta}$ such that $\Delta: y+z=0$.

Proof of the remaining eases are quite similar to that of the first case.

Theorem 3.1. Let $f: \mathbb{R}_{c}^{3} \rightarrow \mathbb{R}_{c}^{3}$ be an isometry. Then there exists a unique $T_{A} \in T(3)$ and $g \in G(D)$ such that $f=T_{A} \circ g$.

Proof. Let $f(O)=A$ where $A=\left(a_{1}, a_{2}, a_{3}\right)$. Define $g=T_{-A} \circ f$. We know that $g$ is an isometry and $g(O)=O$. Thus, $g \in G(D)$ and $f=T_{A} \circ g$ by Proposition 3.9. The proof of uniqueness is trivial. 


\section{REFERENCES}

[1] Chen, B.-Y. and Garay, O. J., An extremal class of conformally flat submanifolds in Euclidean spaces, Acta Math. Hungar., 111(2006), no. 4, 263-303.

[2] Duggal, Krishan L. and Bejancu, A., Lightlike Submanifolds of Semi-Riemannian Manifolds and Applications, Kluwer Academic Publishers, Dordrecht, 1996.

[3] Amstrong, M. A., Groups and Symmetry Springer-Verlag New York Inc., 1988.

[4] Chen, G., Lines and Circles in Taxicab Geometry Master Thesis, Department of Mathematics and Computer Science, Central Missouri State Uni, 1992.

[5] Çolakoğlu, H. B. and Kaya, R., On The Regular Polygons in The Chinese Checker Plane, Appl. Sci. 10 (2008) 29-37.

[6] Gelişgen, Ö., Kaya, R. and Özcan, M., Distance Formulae in the Chinese Checker Space, Int. J. Pure Appl. Math. 26 (2006), no. 1, 35-44.

[7] Gelişgen, Ö. and Kaya, R., Alpha(i) Distance in n-dimensional Space, Appl. Sci. 10 (2008), $88-93$.

[8] Gelişgen, Ö. and Kaya, R., The Taxicab Space Group, Acta Math. Hungar. 122 (2009), no. $1-2,187-200$.

[9] Kaya, R., Gelişgen, Ö., Ekmekçi S. and Bayar, A., Group of Isometries of CC-Plane, Missouri J. Math. Sci. 18 (2006) 221-233.

[10] Kaya, R., Gelişgen, Ö., Ekmekçi S. and Bayar, A., On The Group of Isometries of The Plane with Generalized Absolute Value Metric, Rocky Mountain J. Math. 39 (2009), no. 2, 591-603.

[11] Krause, E. F., Taxicab Geometry Addison - Wesley Publishing Company, Menlo Park, CA, 1975.

[12] Martin, G. E., Transformation Geometry Springer-Verlag New York Inc., 1997.

[13] Schattschneider, D. J., The Taxicab Group, Amer. Math. Monthly 91 (1984) 423-428.

[14] Shen, C. F. C., The Lambda-Geometry Steiner Minimal Tree Problem and Visualization, Phd Thesis, Department of Mathematics and Computer Science, Central Missouri State Uni, 1997.

[15] So, S. S., Recent Development in Metric Geometry, Proceedings of the 5.th National Geometry Symposioum, University of Sakarya, (Sakarya), 2005.

[16] Thompson, A. C., Minkowski Geometry Cambridge University Press, 1996.

[17] http://en.wikipedia.org/wiki/Rotation_matrix

Department of Mathematics and Computer Sciences, Faculty of Science and Arts,

University of Eskisehir Osmangazi, Eskisehir, Turkey.

E-mail address: gelisgen@ogu.edu.tr

Department of Mathematics and Computer Sciences, Faculty of Science and Arts, University of Eskisehir Osmangazi, Eskisehir, Turkey.

E-mail address: rkaya@ogu.edu.tr 